\title{
ERROR BOUNDS FOR SURFACE AREA ESTIMATORS BASED ON CROFTON'S FORMULA
}

\author{
MARKUS KIDERLEN ${ }^{1}$ AND DANiEl MESCHENMOSER ${ }^{2}$ \\ ${ }^{1}$ Department of Mathematical Sciences, University of Aarhus, 8000 Aarhus, Denmark; ${ }^{2}$ Insitute of Stochastics, \\ Ulm University, 89069 Ulm, Germany \\ e-mail: kiderlen@imf.au.dk, daniel.meschenmoser@uni-ulm.de \\ (Accepted September 1, 2009)
}

\begin{abstract}
According to Crofton's formula, the surface area $S(A)$ of a sufficiently regular compact set $A$ in $\mathbb{R}^{d}$ is proportional to the mean of all total projections $p_{A}(u)$ on a linear hyperplane with normal $u$, uniformly averaged over all unit vectors $u$. In applications, $p_{A}(u)$ is only measured in $k$ directions and the mean is approximated by a finite weighted sum $\widehat{S}(A)$ of the total projections in these directions. The choice of the weights depends on the selected quadrature rule. We define an associated zonotope $Z$ (depending only on the projection directions and the quadrature rule), and show that the relative error $\widehat{S}(A) / S(A)$ is bounded from below by the inradius of $Z$ and from above by the circumradius of $Z$. Applying a strengthened isoperimetric inequality due to Bonnesen, we show that the rectangular quadrature rule does not give the best possible error bounds for $d=2$. In addition, we derive asymptotic behavior of the error (with increasing $k$ ) in the planar case. The paper concludes with applications to surface area estimation in design-based digital stereology where we show that the weights due to Bonnesen's inequality are better than the usual weights based on the rectangular rule and almost optimal in the sense that the relative error of the surface area estimator is very close to the minimal error.
\end{abstract}

Keywords: associated zonotope, Crofton formula, digitization, isoperimetric inequality, minimal annulus, perimeter, surface area.

\section{INTRODUCTION}

One common approach to approximate the surface area $S(A)$ of an unknown set $A \subset \mathbb{R}^{d}$ from its digitization is based on a discretization of Crofton's formula. We discuss the worst case error introduced by the discretization of the rotational integral in dependence of the quadrature rule chosen. As the methods apply generally to surface area estimators based on Crofton's formula, we describe them in a general framework and return to its application to digital images in the third section, entitled "Error Bounds for Digital Surface Area Estimators".

Throughout the paper a direction is a vector on the unit sphere $\mathbb{S}^{d-1}$ in $\mathbb{R}^{d}$. If $u$ is a direction, $u^{\perp}$ denotes the linear hyperplane with normal $u$, and $e_{r, u}$ is the straight line with direction $u$ through $r \in u^{\perp}$. Let $A \subset \mathbb{R}^{d}$ be a full-dimensional compact set in the class $\mathscr{U}_{P R}$; (definitions can be found in the next section). A special case of Crofton's formula (Rother and Zähle, 1990) expresses the surface area $S(A)$ of $A$ in terms of the Euler characteristic $\chi$ of linear sections

$$
S(A)=\frac{2}{\gamma_{d}} \int_{\mathbb{S}^{d-1}} \int_{u^{\perp}} \chi\left(A \cap e_{r, u}\right) \mathrm{d} r \mu(\mathrm{d} u) .
$$

Here $\gamma_{d}=\left(2 \kappa_{d-1}\right) /\left(d \kappa_{d}\right)$, where $\kappa_{d}$ is the volume of the $d$-dimensional unit ball, and $\mu$ is the normalized
Haar measure on the unit sphere $\mathbb{S}^{d-1}$; see, e.g., Schneider and Weil (1992, p. 18), but note the different normalization. For sets $A$ that are not full-dimensional, Eq. 1 still holds if its left hand side $S(A)$ is defined in such a way that lower dimensional parts of $A$ are counted twice. The inner integral of Eq. 1,

$$
p_{u}=\int_{u^{\perp}} \chi\left(A \cap e_{r, u}\right) \mathrm{d} r,
$$

is called total projection of $A$ in direction $u$, as it is obtained by measuring the $(d-1)$-volume of the orthogonal projection of $A$ on $u^{\perp}$ with multiplicities. In Eq. 2 the integration is understood with respect to the Lebesgue measure on $u^{\perp}$. If total projections can be determined exactly for finitely many directions $u_{1}, \ldots, u_{k} \in \mathbb{S}^{d-1}$, say, a $k$-point quadrature rule can be used to discretize the outer integral in Eq. 1 and one obtains the approximation

$$
\widehat{S}_{k}^{d}(A)=\frac{2}{\gamma_{d}} \sum_{i=1}^{k} c_{i} p_{u_{i}},
$$

which depends on the choice of weights $c_{1}, \ldots, c_{k} \geq 0$. To assure that the quadrature rule is exact whenever $A$ is a ball, we assume throughout that the weights sum up to 1. If, for example, the rectangular rule is chosen in the planar case, then the weights are proportional to the arc-lengths of the corresponding 
spherical Voronoi cells generated by $\left\{u_{1}, \ldots, u_{k}\right\}$ on $\mathbb{S}^{1}$. This geometric interpretation generalizes readily to higher dimensions. If $\left\{P_{1}, \ldots, P_{k}\right\}$ is the spherical Voronoi tessellation of $\mathbb{S}^{d-1}$ generated by the set of projection directions $\left\{u_{1}, \ldots, u_{k}\right\}$ with $u_{i} \in P_{i}$ then the weights

$$
c_{i}^{V}=\mu\left(P_{i}\right), \quad i=1, \ldots, k,
$$

will be called Voronoi weights associated to $\left\{u_{1}, \ldots, u_{k}\right\}$. These weights are commonly used in applications for $d=2,3$. In the special case where $d=2$ and $u_{1}, \ldots, u_{k}$ are equidistant, the weights for the rectangular quadrature rule (Voronoi weights) coincide with those for the trapezoidal quadrature rule.

The discretization of the spherical integral introduces a bias, which typically depends on the set $A$. We are interested in the worst case behavior. Already Steinhaus (1930) treated the special case where $d=2$, $k$ is even, and $\left\{u_{1}, \ldots, u_{k}\right\}$ forms an equidistant set of points in $\mathbb{S}^{1}$. For $\widehat{S}_{k}^{2}(A)$, given by Eq. 3 with Voronoi weights $c_{1}^{V}, \ldots, c_{k}^{V}$, he derived sharp bounds for the relative error:

$$
\frac{\pi}{k} \frac{\cos (\pi / k)}{\sin (\pi / k)} \leq \frac{\widehat{S}_{k}^{2}(A)}{S(A)} \leq \frac{\pi}{k} \frac{1}{\sin (\pi / k)} .
$$

The left hand side and the right hand side of Eq. 4 are the endpoints of the interval of all possible relative errors, as $A$ varies. Such an interval can be established without the assumption of equidistant directions and in all dimensions. We refer to this interval as error interval in the following.

Using a translative Crofton formula in Section "Error Bounds for $\widehat{S}_{k}^{d}(A)$ ", we will define an originsymmetric convex body $Z \subset \mathbb{R}^{d}$ associated to the discretization, only depending on the projection directions and the quadrature rule. We will show in Lemma 1 that the relative error $\widehat{S}_{k}^{d}(A) / S(A)$ is in a sharp way bounded from below by (a multiple of) the inradius of $Z$ and from above by (a multiple of) the outer radius of $Z$. Thus, the thickness of the minimal annulus of $Z$ is proportional to the length of the error interval and describes the quality of the estimator. Given $k$ projection directions, the quadrature rule (in other words, the values of the associated weights) that minimizes the minimal thickness of $Z$ can typically only be determined numerically. In the planar case, we suggest to bound the thickness of the minimal annulus of $Z$ from above by an isoperimetric deficit using a strengthened isoperimetric inequality due to Bonnesen. This isoperimetric deficit can be minimized with respect to all quadrature rules in closed form. The weights minimizing the isoperimetric deficit will be called Bonnesen weights and are proportional to the lengths of the edges of a polygon circumscribing the unit disk and touching it exactly at the points $u_{1}, \ldots, u_{k}$. We will show that Voronoi weights are not minimizing the length of the error interval by giving an example where the Bonnesen weights yield better error bounds. We will determine the asymptotic behavior (as $k \rightarrow$ $\infty$ ) of the relative error for the Bonnesen weights in Theorem 4. At the end of the second section we will consider the case where the directions $u_{1}, \ldots, u_{k} \in \mathbb{S}^{1}$ are obtained using systematic random sampling on $\mathbb{S}^{1}$. We will show that the coefficient of error of $\widehat{S}_{k}^{2}(A)$ can be bounded from above by a geometric quantity involving $Z$, namely a multiple of the $L_{2}$-distance between $Z$ and its Steiner ball.

In the subsequent section we discuss error bounds for digital surface area estimators. The digitization of $A$ on a randomly translated, rectangular grid will be considered. Asymptotic bounds for the expected value of the estimator for $S(A)$ in the grid will be established. "Asymptotic" relates here to increasing resolution of the grid. The vectors $u_{1}, \ldots, u_{k}$ are chosen as grid directions, i.e., normalized vectors connecting two grid points. These two grid points are usually neighbours and we will consider the 4-, 8- and 16-neighborhood in 2D and the 6- and 26-neighborhood in 3D. For all these settings the Voronoi and Bonnesen weights together with the corresponding in- and circumradii $r$ and $R$, respectively, will be computed analytically, except for the 26 directions in 3D where numerical methods will be used. We will compare the relative errors with the minimal error achieved by numerically optimizing the weights and show that the Bonnesen weights lead, at least in $2 \mathrm{D}$, to smaller errors than the widely used Voronoi weights. We then restrict to quadratic grids in the plane and consider boundary length estimators based on pairs of grid points that are contained in some $(n-1) \times(n-1)$ square of grid cells, $n \geq 2$. This generalizes the case $n=2$, which corresponds to the boundary length estimator based on 8-neighbours. Theorem 7 considers such estimators for general $n \geq 2$ and shows that the asymptotic mean relative error of $\widehat{S}_{k}^{d}(A)$ for Bonnesen weights decreases as $n^{-2}$.

The application of Bonnesen's improved isoperimetric inequality restricts many of the above arguments to the two-dimensional case. In the last section we discuss the possibility of extensions to higher dimensions.

\section{ERROR BOUNDS FOR $\widehat{S}_{k}^{d}(A)$}

A set $A \subset \mathbb{R}^{d}$ is full-dimensional, if its tangent cone at $a$ spans $\mathbb{R}^{d}$ for almost all $a \in A$ with respect 
to $(d-1)$-dimensional Hausdorff measure. If $A$ is topologically regular ( $A$ is the closure of its interior) and convex, it is also full-dimensional. The set $A$ has positive reach if there is an $\varepsilon>0$ such that each point in the $\varepsilon$-neighborhood of $A$ has a unique closest point in $A$.

Throughout the following we assume that $A$ is an element of the family $\mathscr{U}_{P R}$ of all sets in $\mathbb{R}^{d}$ which can be written as a finite union of compact sets $A_{1}, \ldots, A_{m}$ with positive reach such that any intersection $\bigcap_{i \in I} A_{i}$ with $I \subset\{1, \ldots, m\}$ is either empty or a set of positive reach, as well. In particular, convex bodies (nonempty compact convex subsets of $\mathbb{R}^{d}$ ) and polyconvex sets (finite unions of convex bodies) are elements of $\mathscr{U}_{P R}$. For $A \in \mathscr{U}_{P R}$ the surface area measure $S(A, \cdot)$ of order $d-1$ is defined, and Eq. 1 holds with $S(A)=$ $S\left(A, \mathbb{S}^{d-1}\right)$. If $A$ is in addition full-dimensional, $S(A)$ coincides with the usual surface area of $A$. In view of the applications in digital stereology, it is convenient to extend the total projection mapping $v \mapsto p_{v}$ to $\mathbb{R}^{d} \backslash\{o\}$ by positive homogeneity of degree 0 . The translative Crofton formula

$$
p_{v}=\frac{1}{2\|v\|} \int_{\mathbb{S}^{d-1}}|\langle u, v\rangle| S(A, \mathrm{~d} u)
$$

holds for almost all $v \in \mathbb{R}^{d}$; see Rataj (2002, Theorem 2.1 and Theorem 2.3), where $\langle u, v\rangle$ is the usual inner product of $u$ and $v$. If $A$ is polyconvex, Eq. 5 holds for all $v \in \mathbb{R}^{d} \backslash\{o\}$.

If $v_{1}, \ldots, v_{k} \in \mathbb{R}^{d} \backslash\{o\}$ are such that Eq. 5 holds with $v=v_{i}$ for all $i=1, \ldots, k$, then the definition of $\widehat{S}_{k}^{d}(A)$ in combination with Eq. 5 yields

$$
\widehat{S}_{k}^{d}(A)=\frac{1}{\gamma_{d}} \int_{\mathbb{S}^{d-1}} h(u) S(A, \mathrm{~d} u),
$$

with

$$
h:=\sum_{i=1}^{k} \frac{c_{i}}{\left\|v_{i}\right\|}\left|\left\langle v_{i}, \cdot\right\rangle\right|
$$

The key observation is that the integrand $h$ is the support function of a convex body. We refer the reader to Schneider (1993) for relevant notions and concepts in convex geometry and only recall the most important facts here. The support function $h_{K}$ of a convex body $K$ is given by

$$
h_{K}(u)=\max \{\langle x, u\rangle: x \in K\}, \quad u \in \mathbb{S}^{d-1} .
$$

Here and in the following, we consider the support function as a function on the unit sphere. For convex bodies $K$ and $M$ and scalars $\alpha, \beta \geq 0$, we have

$$
\alpha h_{K}+\beta h_{M}=h_{\alpha K \oplus \beta M},
$$

where the Minkowski addition $\oplus$ of sets and the multiplication of a set with a scalar are understood pointwise. We will repeatedly use the monotonicity property,

$$
K \subset M \Longleftrightarrow h_{K} \leq h_{M},
$$

and the fact that the support function $h_{B^{d}}$ of the Euclidean unit ball $B^{d}$ in $\mathbb{R}^{d}$ is the constant 1. Eq. 9 implies in particular that any convex body is uniquely determined by its support function. Consequently, the definition,

$$
\delta_{2}^{2}(K, M):=\int_{\mathbb{S}^{d-1}}\left(h_{K}(u)-h_{M}(u)\right)^{2} \mathrm{~d} u,
$$

for convex bodies $K$ and $M$, gives rise to the so-called $L_{2}$-metric $\delta_{2}(\cdot, \cdot)$ on the family of convex bodies. The support function of the line segment $[-x, x]$ with endpoints $-x$ and $x \in \mathbb{R}^{d}$ is $|\langle x, \cdot\rangle|$. Due to Eq. 8, the function $h$ in Eq. 7 is the support function of a finite sum $Z$ of line segments. Such sets are called zonotopes and play a prominent role in functional analysis, convex and stochastic geometry (see, e.g., Goodey and Weil, 1993 and the references therein). Explicitly, we have

$$
Z=c_{1}\left[-u_{1}, u_{1}\right] \oplus \ldots \oplus c_{k}\left[-u_{k}, u_{k}\right],
$$

with the unit vectors $u_{i}=v_{i} /\left\|v_{i}\right\|$ for $i=1, \ldots, k$. In view of Eq. 6 the approximation $\widehat{S}_{k}^{d}(A)$ can be expressed in terms of the associated zonotope $Z$, as

$$
\widehat{S}_{k}^{d}(A)=\frac{1}{\gamma_{d}} \int_{\mathbb{S}^{d-1}} h_{Z}(u) S(A, \mathrm{~d} u) .
$$

To obtain lower and upper bounds of $\widehat{S}_{k}^{d}(A)$, we have to find maxima and minima of $h_{Z}$. Due to Eq. 9, $r \geq 0$ is the minimum of $h_{Z}$ on $\mathbb{S}^{d-1}$ if and only if $r B^{d}$ is the largest ball contained in Z. Similarly, $R \geq 0$ is the maximum of $h_{Z}$, if and only if $R B^{d}$ is the smallest ball containing $Z$. With these optimal values of $0 \leq r \leq R$, the set $R B^{d} \backslash r B^{d}$ is called the minimal annulus of $Z$. The difference $R-r$ is called the width of the minimal annulus and denoted by $T(Z)$. For later reference we summarize this geometric interpretation for polyconvex sets (for which Eq. 5 holds for arbitrary $v \neq o$ ). As formulations for $\mathscr{U}_{P R}$-sets are obtained in a straightforward manner, we will restrict to polyconvex sets from now on.

Lemma 1 Let $A \subset \mathbb{R}^{d}$ be a polyconvex set with positive surface area, and fix $k \geq 2$ and $v_{1}, \ldots, v_{k} \in$ $\mathbb{R}^{d} \backslash\{o\}$. Let $\gamma_{d}=\left(2 \kappa_{d-1}\right) /\left(d \kappa_{d}\right)$. If $\widehat{S}_{k}^{d}(A)$ is given by Eq. 3, then the sharp bounds

$$
\frac{r}{\gamma_{d}} \leq \frac{\widehat{S}_{k}^{d}(A)}{S(A)} \leq \frac{R}{\gamma_{d}}
$$

for the relative estimation error hold, where $r$ is the smaller and $R$ is larger radius of the minimal annulus of the zonotope $\mathrm{Z}$ given by $\mathrm{Eq} .10$. 
Proof As $R$ is the maximum of $h_{Z}$ on $\mathbb{S}^{d-1}$, it follows from Eq. 11, that

$$
\widehat{S}_{k}^{d}(A) \leq \frac{R}{\gamma_{d}} S(A),
$$

which yields the upper bound. The lower bound follows analoguously from Eq. 11 and the fact that $r$ is the minimum of $h_{Z}$ on $\mathbb{S}^{d-1}$.

That the bounds in the above Lemma are sharp follows from the next example.

Example 2 Fix $k \geq 2, u_{1}, \ldots, u_{k} \in \mathbb{S}^{d-1}$ and weights $c_{1}, \ldots, c_{k} \geq 0$ for a quadrature rule. Define $Z$ according to Eq. 10. Due to symmetry, the ball $r B^{d}$ touches the boundary of $Z$ in at least two antipodal points $r w$ and $-r w, w \in \mathbb{S}^{d-1}$. Let $A$ be a ball of $(d-1)$-volume $1 / 2$ in the hyperplane $w^{\perp}$. (As $A$ is lower dimensional, the proper interpretation of $S(A)$ is twice the $(d-1)$-dimensional Hausdorff measure, so $S(A)=1$.) The surface area measure of $A$ is concentrated on the points $w$ and $-w$, and $h_{Z}$ coincides in both of these directions with $r$, so Eq. 11 implies

$$
\widehat{S}_{k}^{d}(A)=\frac{1}{\gamma_{d}} \int_{\mathbb{S}^{d-1}} r S(A, d u)=\frac{r}{\gamma_{d}} S(A),
$$

and equality holds on the left hand side of Eq. 12.

Fig. 1 illustrates this for $d=2, k=2, u_{1}=(1,0)^{\top}$, $u_{2}=(0,1)^{\top}$ and $c_{1}=c_{2}=1 / 2$. Obviously $A$ is not topologically regular, but it can be approximated by a sequence of topologically regular convex bodies $\left(A_{m}\right)$ in such a way that $\lim _{m \rightarrow \infty} \widehat{S}_{k}^{d}\left(A_{m}\right) / S\left(A_{m}\right)=$ $\widehat{S}_{k}^{d}(A) / S(A)$. This implies that the left hand side of Eq. 12 cannot be improved, even if we restrict considerations to topologically regular sets. To show that the second inequality in Eq. 12 is sharp, a similar argument can be used, if $\pm w$ are directions for which $h_{Z}$ becomes maximal, and thus coincides with $R$.

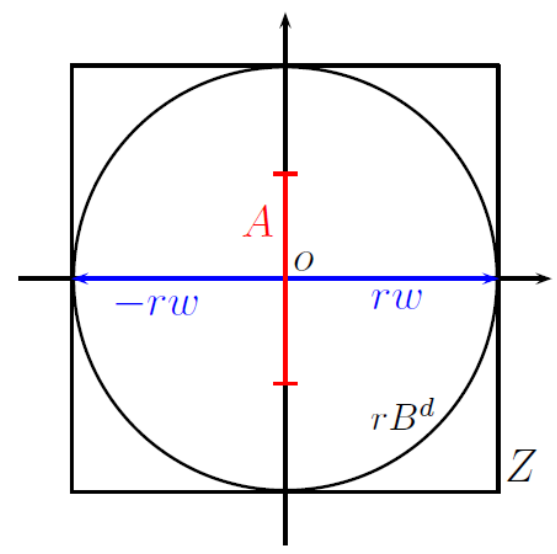

Fig. 1. A possible set $A$ for the case where $Z$ is the unit cube; see Example 2.

\section{THE TWO-DIMENSIONAL CASE}

In the following, we will restrict to the case $d=$ 2, although some of the concepts can be transferred to higher dimensions. As the aim is to minimize the length of the error interval of $\widehat{S}_{k}^{2}(A)$ in Eq. 6, the difference $R-r$ should be as small as possible. This can be achieved by an appropriate choice of the weights $c_{1}, \ldots, c_{k}$. To obtain an exact value for the integral in Eq. 6 in the case where $A$ is a disk, we must assume that the weights sum up to one. It follows from Schneider (1993), that $c_{1}+\ldots+c_{k}=1$ is equivalent to the condition that the zonotope $Z$ given by Eq. 10 has perimeter 4 . Let $\mathscr{Z}$ be the family of all zonotopes that can be written as sum of line-segments parallel to given unit vectors $u_{1}, \ldots, u_{k}$. Let $\mathscr{Z}_{4}$ be the family of those $Z \in \mathscr{Z}$ that have perimeter 4 . We are therefore faced with the problem of finding a zonotope $Z^{*} \in \mathscr{Z}_{4}$ that satisfies

$$
T\left(Z^{*}\right)=\min \left\{T(Z): Z \in \mathscr{Z}_{4}\right\} .
$$

If

$$
Z^{*}=c_{1}^{*}\left[-u_{1}, u_{1}\right] \oplus \ldots \oplus c_{k}^{*}\left[-u_{k}, u_{k}\right],
$$

then $c_{1}^{*}, \ldots, c_{k}^{*} \geq 0$ are the best weights in Eq. 3, in the sense that among all weights summing up to one they yield the shortest interval of possible relative errors. A solution $Z^{*}$ of the optimization problem in Eq. 13 always exists due to a compactness argument based on the Blaschke selection theorem.

For asymptotic results, it is enough to replace the objective function in Eq. 13 by a simpler one. Bonnesen (1929) improved the isoperimetric inequality for an arbitrary planar convex body $K$, stating that

$$
\frac{S^{2}(K)}{4 \pi}-V(K) \geq \frac{\pi}{4} T^{2}(K),
$$

where $S(K)$ and $V(K)$ are perimeter and area of $K$, respectively. For $K=Z \in \mathscr{Z}_{4}$ we have $S(Z)=4$ and the left hand side of Eq. 14 is minimal for the zonotope $\widetilde{Z} \in$ $\mathscr{Z}_{4}$ that has the greatest area. According to a classical result of Lindelöf (1869), $\widetilde{Z}$ is characterized among all zonotopes in $\mathscr{Z}_{4}$ by the fact that it circumscribes a circle. Due to origin-symmetry, this circle is the incircle of $\widetilde{Z}$, centered at the origin, and with radius $\widetilde{r}_{k}$. This allows an explicit construction of $\widetilde{Z}$. Up to scaling with the factor $1 / \widetilde{r}_{k}$ the zonotope $\widetilde{Z}$ coincides with the polytope

$$
\widetilde{P}:=\bigcap_{i=1}^{k}\left\{x \in \mathbb{R}^{2}:\left|\left\langle u_{i}, x\right\rangle\right| \leq 1\right\},
$$


obtained by intersecting all supporting half-planes of the unit disk with outer normal in $\left\{ \pm u_{1}, \ldots, \pm u_{k}\right\}$. We now assume without loss of generality that the vectors $u_{1}, \ldots, u_{k}$ all are located on the positive half-sphere $\{(\cos \varphi, \sin \varphi): 0 \leq \varphi<\pi\}$ and are ordered according to increasing angles. We write $\Varangle(u, v) \in[0, \pi]$ for the (smaller) angle between the unit vectors $u$ and $v$. Let $\alpha_{i}$ be the outer angle of the vertex between the $i$ th and the $(i+1)$ st edge (i.e., $\alpha_{i}$ is the angle of the normal cone at this vertex, in other words $\pi-\alpha_{i}$ is the usual inner angle; see Fig. 2). Explicitly, we have

$$
\alpha_{i}= \begin{cases}\Varangle\left(u_{i}, u_{i+1}\right), & i=1, \ldots, k-1, \\ \pi-\Varangle\left(u_{1}, u_{k}\right), & i=k,\end{cases}
$$

as the normal of the $(k+1)$ st edge is $-u_{1}$.

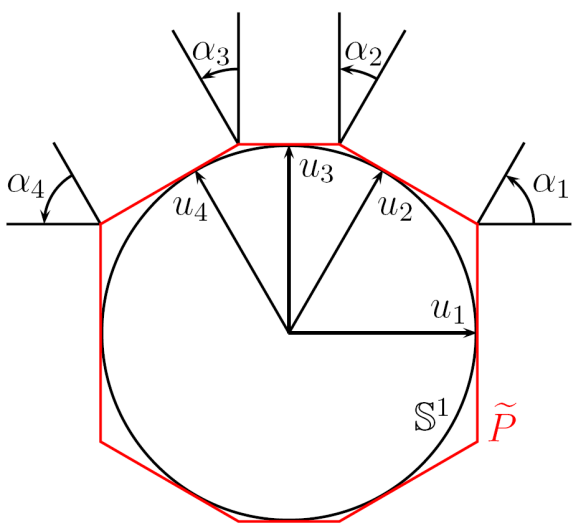

Fig. 2. Construction of the angles $\alpha_{i}$ and the polytope $\widetilde{P}$ given by $E q .15$ with $k=4$.

As the length of the $i$ th edge of $\widetilde{P}$ is $\tan \left(\alpha_{i} / 2\right)+$ $\tan \left(\alpha_{i-1} / 2\right)$ and $S(\widetilde{Z})=4$, we obtain

$$
\widetilde{Z}=\widetilde{c}_{1}\left[-u_{1}, u_{1}\right] \oplus \ldots \oplus \widetilde{c}_{k}\left[-u_{k}, u_{k}\right],
$$

with

$$
\widetilde{c}_{i}=\widetilde{r}_{k} \frac{\tan \left(\alpha_{i} / 2\right)+\tan \left(\alpha_{i-1} / 2\right)}{2},
$$

and

$$
\widetilde{r}_{k}=\left(\sum_{i=1}^{k} \tan \left(\alpha_{i} / 2\right)\right)^{-1},
$$

where we have put $\alpha_{0}=\alpha_{k}$. This and $V(\widetilde{Z})=2 \widetilde{r}_{k}$ was also derived by Knebelman (1941). The weights $\widetilde{c_{i}}$ are based on the application of Bonnesen's inequality Eq. 14 and will be called Bonnesen weights in the following. The $i$ th weight $\widetilde{c}_{i}$ is the relative length of the $i$ th edge of the polygon with facet normals $\pm u_{i}$ which circumscribes a circle of radius $\widetilde{r}_{k}$. The outer radius $\widetilde{R}_{k}$ of $\widetilde{Z}$ is the largest distance of a vertex of $\widetilde{Z}$ from the origin, and this is

$$
\widetilde{R}_{k}=\widetilde{r}_{k} \max _{i=1}^{k} \frac{1}{\cos \left(\alpha_{i} / 2\right)} .
$$

Summarizing, we have shown the following. If $\widehat{S}_{k}^{2}(A)$ is an estimator of $S(A)>0$ given by Eq. 6 with the Bonnesen weights $c_{i}=\widetilde{c}_{i}, i=1, \ldots, k$, from Eq. 17, then the relative errors obey

$$
\frac{\pi}{2} \widetilde{r}_{k} \leq \frac{\widehat{S}_{k}^{2}(A)}{S(A)} \leq \frac{\pi}{2} \widetilde{R}_{k} .
$$

These error bounds are sharp; see Example 2.

It should be noted that Eq. 14 is always a strict inequality unless $K$ is a disk. As Eq. 14 is used for zonotopes here, this approach will not necessarily lead to the optimal choice of the weights. However, the choice may be better than choices for the weights motivated by usual quadrature rules.

Example 3 We consider the integrand in Eq. 6 with $k=3$ and the directions $u_{i}=\left(\cos \left(\varphi_{i}\right), \sin \left(\varphi_{i}\right)\right), i=$ $1,2,3$, where

$$
\varphi_{1}=0, \quad \varphi_{2}=\frac{\pi}{16}, \quad \varphi_{3}=\frac{\pi}{8} .
$$

As mentioned before, the rectangular quadrature rule leads to the Voronoi weights $c_{i}^{V}$ : The ith weight is the normalized length of the Voronoi arc corresponding to $u_{i}$ (arc in $\mathbb{S}^{1}$ of all points closer to $u_{i}$ than to any other point in $\left.\left\{ \pm u_{1}, \pm u_{2}, \pm u_{3}\right\}\right)$. This gives

$$
c_{i}^{V}=\frac{\varphi_{i+1}-\varphi_{i-1}}{2 \pi},
$$

where we assumed $\pi$-periodicity. For the present example, we obtain

$$
\mathbf{c}^{V}=\left(\frac{15}{32}, \frac{1}{16}, \frac{15}{32}\right)
$$

and the corresponding zonotope $Z_{V}$ has inradius $r_{V} \approx 0.18290$ and circumradius $R_{V} \approx 0.98199$; see Fig. 3. Thus, the width of the minimal annulus is approximately 0.79909 .

Using instead the Bonnesen weights yields approximately

$$
\widetilde{\mathbf{c}}=(0.49057,0.01885,0.49057),
$$

leading to the inradius $\widetilde{r} \approx 0.191412$ and circumradius $\widetilde{R} \approx 0.981147$, respectively. The width of the minimal annulus is now approximately 0.789735 , which is an improvement of about $1 \%$. 


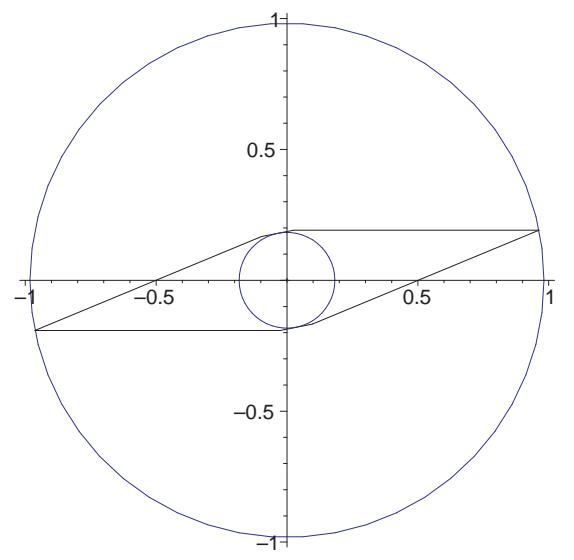

Fig. 3. The zonoid from Example 3 associated to Voronoi weights together with its minimal annulus.

To formulate an asymptotic result, we have to specify how close the set of the directions $u_{1}, \ldots, u_{k}$ is to a set of equidistant directions. Following Gardner et al. (2006), we introduce the symmetrized spread $\Delta_{k}^{*}$ of $u_{1}, \ldots, u_{k}$ by

$$
\Delta_{k}^{*}=\max _{u \in \mathbb{S}^{1}} \min _{1 \leq i \leq k} \min \left\{\left\|u-u_{i}\right\|,\left\|u-\left(-u_{i}\right)\right\|\right\} .
$$

Geometrically, $\Delta_{k}^{*}$ is the maximal distance of a unit vector from the set $\left\{ \pm u_{1}, \ldots, \pm u_{k}\right\}$. In particular, $\left\{ \pm u_{1}, \ldots, \pm u_{k}\right\}$ is a $\Delta_{k}^{*}$-net in $\mathbb{S}^{1}$. For $\alpha_{i}$ defined by Eq. 16, we have

$$
2 \sin \frac{\alpha_{i}}{4} \leq \Delta_{k}^{*}, \quad i=1, \ldots, k
$$

The following theorem shows that the choice $c_{i}=$ $\widetilde{c}_{i}$ leads to a relative error of $\widehat{S}_{k}^{2}(A)$ that depends quadratically on $\Delta_{k}^{*}$. Here we only consider sampling sets $\left\{u_{1}, \ldots, u_{k}\right\}$ such that every closed sub-arc of $\mathbb{S}^{1}$ of length $\pi / 2$ contains at least one point of $\left\{ \pm u_{1}, \ldots, \pm u_{k}\right\}$. Equivalently, $\Delta_{k}^{*} \leq \sqrt{2-\sqrt{2}}$.

Theorem 4 Let $A \subset \mathbb{R}^{2}$ be a polyconvex set with positive perimeter. Let $k \geq 2$ and $\left\{v_{1}, \ldots, v_{k}\right\} \subset \mathbb{R}^{2} \backslash$ $\{0\}$ such that the symmetrized spread of the vectors $u_{i}=v_{i} /\left\|v_{i}\right\|, i=1, \ldots, k$, is $\Delta_{k}^{*} \leq \sqrt{2-\sqrt{2}}$. If $\widehat{S}_{k}^{2}(A)$ in Eq. 3 is calculated using the Bonnesen weights $c_{i}=\widetilde{c}_{i}$, $i=1, \ldots, k$, from $E q .17$, then the relative error obeys

$$
\left|\frac{\widehat{S}_{k}^{2}(A)-S(A)}{S(A)}\right| \leq \frac{\pi^{2}}{3}\left(\Delta_{k}^{*}\right)^{2} .
$$

Proof From Eq. 20 we get

$$
\pi \widetilde{r}_{k}-2 \leq 2\left(\frac{\widehat{S}_{k}^{2}(A)-S(A)}{S(A)}\right) \leq \pi \widetilde{R}_{k}-2 .
$$

We estimate the left hand side of Eq. 23. In view of Eq. 21, we have $\alpha_{i} / 2 \leq 2 \arcsin \left(\Delta_{k}^{*} / 2\right) \leq \pi / 4$ for all $i=1, \ldots, k$. Taylor's theorem implies

$\tan \left(\alpha_{i} / 2\right) \leq \alpha_{i} / 2+c^{\prime}\left(\alpha_{i} / 2\right)^{3}, \quad$ for all $i=1, \ldots, k$,

where $c^{\prime}=8 / 3$ is the third derivative of $\tan (x) / 3$ ! evaluated at $\pi / 4$. Relations 18,21 and $\arcsin (x) \leq \frac{\pi}{2} x$, $0 \leq x \leq 1$, imply that

$$
\pi \widetilde{r}_{k} \geq \frac{\pi}{\sum_{i=1}^{k}\left(\frac{\alpha_{i}}{2}\left(1+c^{\prime}\left(\frac{\alpha_{i}}{2}\right)^{2}\right)\right)} \geq \frac{2}{1+\frac{c^{\prime} \pi^{2}}{4}\left(\Delta_{k}^{*}\right)^{2}},
$$

and this gives

$$
\pi \widetilde{r}_{k}-2 \geq-\frac{2 \pi^{2}}{3}\left(\Delta_{k}^{*}\right)^{2}
$$

The right hand side of Eq. 23 can be estimated in an even easier way using the fact that the perimeter of the incircle of $\widetilde{Z}$ is bounded by $S(\widetilde{Z})=4$ and hence $\widetilde{r}_{k} \leq 2 / \pi$. Together with Eq. 19 this gives

$$
\pi \widetilde{R}_{k}-2 \leq \frac{2}{1-\left(\Delta_{k}^{*}\right)^{2}}\left(\Delta_{k}^{*}\right)^{2} \leq \frac{2}{\sqrt{2}-1}\left(\Delta_{k}^{*}\right)^{2},
$$

as $\Delta_{k}^{*} \leq \sqrt{2-\sqrt{2}}$. Putting things together we arrive at

$$
\left|\frac{\widehat{S}_{k}^{2}(A)-S(A)}{S(A)}\right| \leq c\left(\Delta_{k}^{*}\right)^{2}
$$

with $c=\max \left\{\pi^{2} / 3,1 /(\sqrt{2}-1)\right\}=\pi^{2} / 3$.

Using the fact that Voronoi weights deviate only slightly from Bonnesen weights as $k$ increases, it can be shown that the same order of convergence also holds for the relative error of $\widehat{S}_{k}^{2}(A)$ if the estimator is based on Voronoi weights. The example of equidistant sampling shows that quadratic behavior is the best possible.

Example 5 Consider the special case where $u_{1}, \ldots, u_{k}$ are equidistant on the upper half circle, meaning that $u_{i}=(\cos (i \pi / k), \sin (i \pi / k)), i=1, \ldots, k$. Hence

$$
\Delta_{k}^{*}=2 \sin \frac{\pi}{4 k} \sim \frac{\pi}{2 k}, \quad k \rightarrow \infty .
$$

By symmetry arguments, the weights leading to the minimal width of the corresponding minimal annulus must all be equal and thus $c_{1}=\ldots=c_{k}=1 / k$ and $c_{i}=\widetilde{c}_{i}$ for $i=1, \ldots, k$. According to Eqs. 18 and 19, the inner and outer radii of the associated zonotope are

$$
\widetilde{r}_{k}=\left(k \tan \left(\frac{\pi}{2 k}\right)\right)^{-1}
$$


and

$$
\widetilde{R}_{k}=\widetilde{r}_{k}\left(\cos \left(\frac{\pi}{2 k}\right)\right)^{-1}=\left(k \sin \left(\frac{\pi}{2 k}\right)\right)^{-1},
$$

$c f$. Eq. 4. Therefore, the width of the minimal annulus is

$$
\widetilde{R}_{k}-\widetilde{r}_{k}=\frac{\pi}{4} k^{-2}+O\left(k^{-4}\right), \quad k \rightarrow \infty
$$

This shows that $\widetilde{R}_{k}-\widetilde{r}_{k}$, and thus the relative worst case error, are of order $\left(\Delta_{k}^{*}\right)^{2}$.

Instead of using the above geometric arguments to obtain asymptotics for the worst case error, one might also use methods from optimum quantization (see Gruber, 2004). Among other important applications, this theory yields asymptotic minimum errors of numerical integration for classes of Hölder continuous functions. As the function $g_{u}: v \mapsto|\langle v, u\rangle|$, and hence the function $p_{v}$ in Eq. 5 are Lipschitz continuous, optimum quantization gives an upper bound for the worst case error depending linearly on $\Delta_{k}$. This suboptimal rate is due to the fact that the class of Hölder continuous functions with Hölder exponent 1 is considerably larger than its subspace spanned by $\left\{g_{u}: u \in \mathbb{S}^{1}\right\}$.

\section{A SEMI-RANDOMIZED APPROACH}

The associated zonotope for quadrature rules can also be used in the context of a semirandomized approach, which generalizes systematic random sampling designs. The idea of this design based approach is to evaluate the total projections of the randomly rotated set $\vartheta A$ in $k$ directions. In other words, given $k$ vectors $v_{1}, \ldots, v_{k} \in \mathbb{S}^{d-1}$ and weights $c_{1}, \ldots, c_{k}$, the estimator for $S(A)$ is defined by

$$
\widehat{S}_{k}^{d}(\vartheta A)=\frac{2}{\gamma_{d}} \sum_{i=1}^{k} c_{i} p_{\vartheta-1} v_{i}
$$

where $\vartheta$ is a random rotation whose distribution is the normalized Haar measure on the compact group $S O_{d}$ of proper rotations. Clearly, Eq. 24 defines a random variable and Crofton's formula implies that this variable is an unbiased estimator for $S(A)$. In particular, if $d=2$ and the set $\left\{ \pm v_{1}, \ldots, \pm v_{k}\right\}$ is equidistant in $\mathbb{S}^{1}$, the estimator $\widehat{S}_{k}^{d}(\vartheta A)$ in Eq. 24 is the one obtained from systematic random sampling. Moran (1966) considered this special case and gave worst case bounds for the variance of $\widehat{S}_{k}^{d}(\vartheta A)$. His approach allows a geometric interpretation which is not restricted to the planar setting: Let $Z$ be, again, the zonotope associated to a fixed quadrature rule. From Eq. 11 and the unbiasedness of the estimator, we get

$$
\begin{aligned}
\operatorname{Var}\left(\widehat{S}_{k}^{d}(\vartheta A)\right)=\mathbb{E}_{\vartheta}\left(\widehat{S}_{k}^{d}(\vartheta A)-S(A)\right)^{2} \\
\quad=\gamma_{d}^{-2} \mathbb{E}_{\vartheta}\left(\int_{\mathbb{S}^{d-1}}\left(h_{Z}(\vartheta u)-\gamma_{d}\right) S(A, \mathrm{~d} u)\right)^{2}
\end{aligned}
$$

Hence

$$
\begin{aligned}
\operatorname{Var}\left(\widehat{S}_{k}^{d}(\vartheta A)\right)= \\
\quad \gamma_{d}^{-2} \int_{\mathbb{S}^{d-1}} \int_{\mathbb{S}^{d-1}} J(u, v) S(A, \mathrm{~d} u) S(A, \mathrm{~d} v)
\end{aligned}
$$

where

$$
J(u, v)=\mathbb{E}_{\vartheta}\left(\left(h_{Z}(\vartheta u)-\gamma_{d}\right)\left(h_{Z}(\vartheta v)-\gamma_{d}\right)\right)
$$

Hölder's inequality implies that

$$
\begin{aligned}
J(u, v) \leq & \left(\mathbb{E}_{\vartheta}\left(h_{Z}(\vartheta u)-\gamma_{d}\right)^{2}\right)^{1 / 2} \\
& \times\left(\mathbb{E}_{\vartheta}\left(h_{Z}(\vartheta v)-\gamma_{d}\right)^{2}\right)^{1 / 2} \\
= & \int_{\mathbb{S}^{d-1}}\left(h_{Z}(u)-\gamma_{d}\right)^{2} \mu(\mathrm{d} u)
\end{aligned}
$$

Hence

$$
\operatorname{Var}\left(\widehat{S}_{k}^{d}(\vartheta A)\right) \leq \frac{S(A)^{2}}{\gamma_{d}^{2} \varpi_{d}} \delta_{2}^{2}\left(Z, \gamma_{d} B^{d}\right)
$$

where $\delta_{2}(\cdot, \cdot)$ denotes the $L_{2}$-metric defined earlier. This inequality is sharp, as equality holds here for example whenever $A$ is a set of dimension $d-$ 1. The quadrature rule is exact when $A=B^{d}$ and thus $\widehat{S}_{k}^{d}\left(B^{d}\right)=d \kappa_{d}$, which implies that $2 \gamma_{d}$ is the mean width $b(Z)$ of $Z$ and $\gamma_{d} B^{d}$ is the Steiner ball of Z; see Schneider (1993, p. 353). Hence, the coefficient of variation $\sqrt{\operatorname{Var}\left(\widehat{S}_{k}^{d}(\vartheta A)\right)} / S(A)$ is bounded from above by a multiple of the $L_{2}$ distance of $Z$ to its Steiner ball. Again, a geometric inequality (Groemer, 1990) would allow to give an upper bound of the right hand side of Ineq. 26. However, a more direct evaluation is possible and was carried out by Janáček (2001). In particular he found optimal weights $c_{1}, \ldots, c_{k}$ to minimize the variance in the case of a "totally anisotropic object", i.e., a set $A$ contained in a hyperplane. The optimal weights are obtained by inverting the covariance matrix of $\left(p_{\vartheta^{-1} u_{1}}, \ldots, p_{\vartheta^{-1} u_{k}}\right)$. 


\section{ERROR BOUNDS FOR DIGITAL SURFACE AREA ESTIMATORS}

In this section we consider digitizations of a topologically regular polyconvex set $A \subset \mathbb{R}^{d}$ on rectangular grids and assume that the directions $u_{i}$ are given as normalized difference vectors of grid points, which usually are neigbours. For example, we consider the common 4- and 8-neighborhoods in the plane. We compute Voronoi- and Bonnesen-weights together with the associated in- and circumradii explicitly and give asymptotic error bounds for $\widehat{S}(A)$ for increasing resolution of the digitization.

To digitize $A$ we consider the rectangular point grid $\mathbb{G}=\zeta_{1} \mathbb{Z} \times \zeta_{2} \mathbb{Z} \times \ldots \times \zeta_{d-1} \mathbb{Z} \times \mathbb{Z}$, where $\zeta_{1}, \ldots, \zeta_{d-1}>0$ are the grid distances in the directions of the axes, and we used the grid distance in the last coordinate direction as unit. A grid cell is any $d$-dimensional cuboid $z+\left[0, \zeta_{1}\right] \times \ldots \times\left[0, \zeta_{d-1}\right] \times$ $[0,1], z \in \mathbb{G}$. Motivated by design based stereology, we consider digitizations of $A$ by a randomly translated grid. With the random variable $\xi$, uniformly distributed in an arbitrarily chosen grid cell, the random grid $\xi+\mathbb{G}$ is a stationary random closed set and is called a stationary grid in Kiderlen and Rataj (2006).

In order to increase resolution, we scale the grid by a factor $t>0$ and denote the digitization of $A$ in the scaled grid $t(\xi+\mathbb{G})$ by $\Delta_{t}(A)$. Let $Q$ be a non-empty compact set, called the sampling element. We assume that each grid point $x \in t(\xi+\mathbb{G})$ is the center of a small sampling window $x+t Q$, which can be thought of as a pixel or voxel. The pixel digitization consists of all grid points $x$ for which this sampling window $x+t Q$ hits the set $A$. Hence $\Delta_{t}(A)=(A \oplus t \check{Q}) \cap t(\xi+\mathbb{G})$, where $\check{Q}$ is the reflection of $Q$ at the origin. For $Q=\{o\}$ the pixel digitization reduces to the Gauss digitization (sometimes called hit-or-miss digitization) containing all points of the scaled grid in A. All results on error bounds in this section will be stated for the pixel digitization and therefore also hold for the Gauss digitization. In image processing, the term digitization often denotes the union of pixels (grid cells) which are centered at grid points in $\Delta_{t}(A)$. As such pixel unions are in one-to-one correspondence with the unions of their centers, one may equivalently consider digitizations as subsets of the grid, and we will do so througout the paper.

We fix a set $A$ and estimate its surface area $S(A)$ from the information available in its digitization $\Delta_{t}(A)$ using a discretized Crofton formula; cf. Ohser and Mücklich (2000). We will focus on the asymptotic error of this estimator when the grid spacing gets finer, i.e., when the digitized set $\Delta_{t}(A)$ becomes a better approximation of the original set $A$. The function $p_{v}$ given by Eq. 2 can easily be estimated from the digitized set $\Delta_{t}(A)$ by comparing the values of neighboring points, if $v$ is a grid point. For any vector $v \in \mathbb{G} \backslash\{o\}$ such an estimator is given by

$$
\begin{aligned}
& \widehat{p}_{v}(t)= \\
& \quad \frac{t^{d-1}}{\|v\|} \#\left\{x \in t(\xi+\mathbb{G}): x \in \Delta_{t}(A), x+t v \notin \Delta_{t}(A)\right\} .
\end{aligned}
$$

This estimator counts the number of points $x$ in the digitized set $\Delta_{t}(A)$ such that $x+t v$ does not lie in the digitization of $A$. From Kiderlen and Rataj (2006, Theorem 5) it follows that this estimator is asymptotically unbiased, i.e.,

$$
\lim _{t \searrow 0} \mathbb{E} \widehat{p}_{v}(t)=p_{v}
$$

Having chosen $k$ vectors $v_{1}, \ldots, v_{k} \in \mathbb{G}$ and scalars $c_{1}, \ldots, c_{k} \geq 0$, one can define

$$
\widehat{S}_{k}^{d}(A ; t)=\frac{2}{\gamma_{d}} \sum_{i=1}^{k} c_{i} \widehat{p}_{v_{i}}(t),
$$

and it follows from Eq. 27 that $\widehat{S}_{k}^{d}(A ; t)$ is an asymptotically unbiased estimator for $\widehat{S}_{k}^{d}(A)$, as $t \searrow 0$. Note that the estimator given by Eq. 28 can be calculated from the knowledge of the digitization $\Delta_{t}(A)$ of $A$ alone. $\widehat{S}_{k}^{d}(A ; t)$ behaves approximately like a discretized Crofton integral, when $t$ is small. Thus, the methods and results of the previous section can be applied to obtain asymptotic error bounds.

To illustrate this approach we discretize the Crofton integral using only directions parallel to the coordinate axes: in $\mathbb{R}^{d}$ we choose the $2 d$ grid points $v_{i}=-v_{d+i}=\zeta_{i} e_{i}, i=1, \ldots, d-1$ and $v_{d}=-v_{2 d}=e_{d}$ where $e_{i}$ denotes the $i$ th unit vector. Due to symmetry, the weights leading to a minimal error interval are all equal, $c_{i}=1 /(2 d), i=1, \ldots, 2 d$, and coincide with the Voronoi weights. The zonotope $Z$ defined in Eq. 10 is given by $Z=[-1 / d, 1 / d]^{d}$ and it has inradius $r=1 / d$ and circumradius $R=1 / \sqrt{d}$. Lemma 1 and the asymptotic unbiasedness of $\widehat{S}_{2 d}^{d}(A ; t)$ imply

$$
\frac{1}{d \gamma_{d}} \leq \lim _{t \searrow 0} \frac{\mathbb{E} \widehat{S}_{2 d}^{d}(A ; t)}{S(A)} \leq \frac{1}{\sqrt{d} \gamma_{d}} .
$$

In the planar case we have $\gamma_{2}=2 / \pi$ and

$$
0.785 \approx \frac{\pi}{4} \leq \lim _{t \searrow 0} \frac{\mathbb{E} \widehat{S}_{4}^{2}(A ; t)}{S(A)} \leq \frac{\pi}{4} \sqrt{2} \approx 1.111,
$$


which means the asymptotic relative error is $21.5 \%$ in the worst case. In three dimensions, the asymptotic relative error is at most $33.3 \%$ as $\gamma_{3}=1 / 2$ and

$$
0.667 \approx \frac{2}{3} \leq \lim _{t \searrow 0} \frac{\mathbb{E} \widehat{S}_{6}^{3}(A ; t)}{S(A)} \leq \frac{2}{3} \sqrt{3} \approx 1.155 .
$$

Due to Stirling's formula we have $\sqrt{d} \gamma_{d} \rightarrow \sqrt{2 / \pi}$ as $d \rightarrow \infty$. As $\sqrt{d} \gamma_{d}$ is decreasing in $d$, we have

$$
0 \leq \lim _{t \searrow 0} \frac{\mathbb{E} \widehat{S}_{2 d}^{d}(A ; t)}{S(A)} \leq \sqrt{\frac{\pi}{2}} \approx 1.253,
$$

for all $d$, where $\sqrt{\pi / 2}$ is the best upper bound that holds for arbitrary dimension $d$. We do not obtain a non-trivial uniform lower bound, as there are $d \in \mathbb{N}$ and sets $A$ in $\mathbb{R}^{d}$ with $S(A)=1$, but such that $\widehat{S}_{2 d}^{d}(A)$ is arbitrarily close to 0 . Due to the large worst case error even in low dimensions, the above choice of grid points is not recommended for practical applications. Instead, a larger number of grid points should be used. On the other hand, the estimator of $S(A)$ in Eq. 28 is based on asymptotic considerations and becomes less reliable when the lengths of the vectors $v_{i}$ are large. One would therefore restrict to vectors with bounded length, i.e., vectors contained in a small Euclidean disk around the origin. For computational reasons it is easier to replace the Euclidean disk by a disk with respect to the maximum norm. To be specific, we choose $\left\{v_{1}, \ldots, v_{k}\right\}$ in the set

$$
\begin{aligned}
& \mathscr{V}_{n}^{(d)}:=\left[-\zeta_{1}(n-1), \zeta_{1}(n-1)\right] \times \ldots \\
\times & {\left[-\zeta_{d-1}(n-1), \zeta_{d-1}(n-1)\right] \times[-(n-1), n-1] \backslash\{o\} . }
\end{aligned}
$$

For $n=2$, the most common choice in applications, we have

$$
\begin{aligned}
\mathscr{V}_{2}^{(d)}= & \left\{-\zeta_{1}, 0, \zeta_{1}\right\} \times \ldots \\
& \times\left\{-\zeta_{d-1}, 0, \zeta_{d-1}\right\} \times\{-1,0,1\} \backslash\{o\},
\end{aligned}
$$

and $k=\# \mathscr{V}_{2}^{(d)}=3^{d}-1$. We determine asymptotic worst case errors for $n=2$ and $n=3$ in the planar case and for $n=2$ in dimension $d=3$.

\section{THE TWO-DIMENSIONAL CASE}

In the planar case for $n=2$, the $k=3^{2}-1=$ 8 grid points of the set in Eq. 29 are just the 8-neighbours of the origin, and the corresponding directions are parallel to the edges and diagonals of a grid cell. Both the Voronoi weights and the Bonnesen weights can be computed analytically. It turns out that the Voronoi weights and the Bonnesen weights do not coincide, and the corresponding in- and circumradii are different. In the following let $\beta=$ $\arccos \left(\zeta_{1} / \sqrt{\zeta_{1}^{2}+1}\right)$. For the Voronoi case, the inradius $r_{\text {Vor }}\left(\zeta_{1}\right)$ and the circumradius $R_{\text {Vor }}\left(\zeta_{1}\right)$ are given by

$$
r_{\text {Vor }}\left(\zeta_{1}\right)= \begin{cases}\frac{1}{\pi} \beta+\frac{\zeta_{1}}{2 \sqrt{\zeta_{1}^{2}+1}}, & \text { if } \zeta_{1} \leq 1 \\ \frac{1}{2}-\frac{1}{\pi} \beta+\frac{1}{2 \sqrt{\zeta_{1}^{2}+1}}, & \text { otherwise }\end{cases}
$$

and

$$
R_{\operatorname{Vor}}\left(\zeta_{1}\right)=\left\{\begin{array}{c}
\frac{1}{\pi}\left[\beta^{2}+\left(\frac{\pi}{2}-\beta+\frac{\pi}{2 \sqrt{\zeta_{1}^{2}+1}}\right)^{2}\right]^{1 / 2}, \\
\text { if } \zeta_{1} \leq 1, \\
\frac{1}{\pi}\left[\left(\beta+\frac{\pi}{2} \frac{\zeta_{1}}{\sqrt{\zeta_{1}^{2}+1}}\right)^{2}+\left(\frac{\pi}{2}-\beta\right)^{2}\right]^{1 / 2}, \\
\text { otherwise, }
\end{array}\right.
$$

respectively. In the Bonnesen case the inradius $r_{\mathrm{Bon}}\left(\zeta_{1}\right)$ is given by

$$
r_{\mathrm{Bon}}\left(\zeta_{1}\right)=\zeta_{1}\left(2 \sqrt{\zeta_{1}^{2}+1}\left(\zeta_{1}+1\right)-2\left(\zeta_{1}^{2}+1\right)\right)^{-1}
$$

and the circumradius $R_{\mathrm{Bon}}\left(\zeta_{1}\right)$ is given by

$$
R_{\mathrm{Bon}}\left(\zeta_{1}\right)=\left\{\begin{array}{c}
r_{\mathrm{Bon}}\left(\zeta_{1}\right) \sqrt{2}\left(1+\left(\frac{1}{\zeta_{1}^{2}}+1\right)^{-1 / 2}\right)^{-1 / 2} \\
\text { if } \zeta_{1} \leq 1 \\
r_{\mathrm{Bon}}\left(\zeta_{1}\right) \sqrt{2}\left(1+\left(\zeta_{1}^{2}+1\right)^{-1 / 2}\right)^{-1 / 2}, \\
\text { otherwise. }
\end{array}\right.
$$

Neither the Voronoi nor the Bonnesen weights are optimal in the sense that they minimize the length of the asymptotic error interval. The optimal weights were found by numerically solving a constrained minimization problem using MATLAB. It turns out that the Bonnesen weights are very close to the optimum as can be seen from Fig. 4 which shows the thickness of the minimal annulus for Voronoi, Bonnesen, and optimized weights, respectively, depending on $\zeta_{1}>0$. Interchanging the $x$ - and the $y$-axis, if necessary, we may restrict to $\zeta_{1} \leq 1$. 


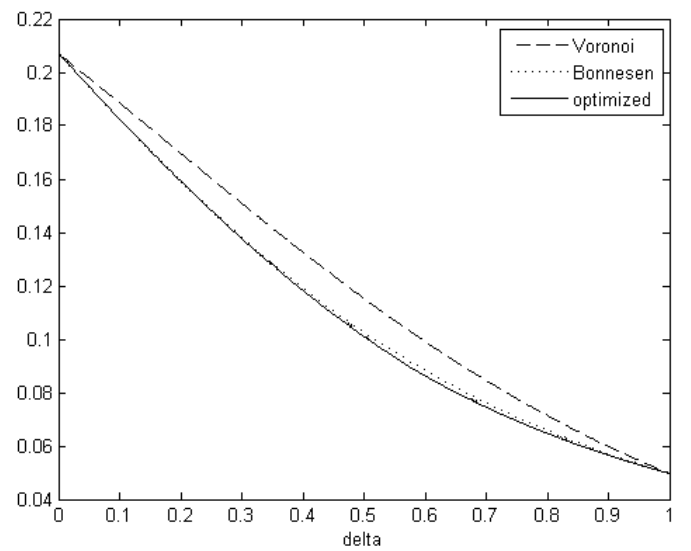

Fig. 4. Thickness of the minimal annulus for eight directional vectors on a rectangular grid in $2 D$ depending on $\zeta_{1}$ for Voronoi, Bonnesen, and optimized weights, respectively (see the text for details).

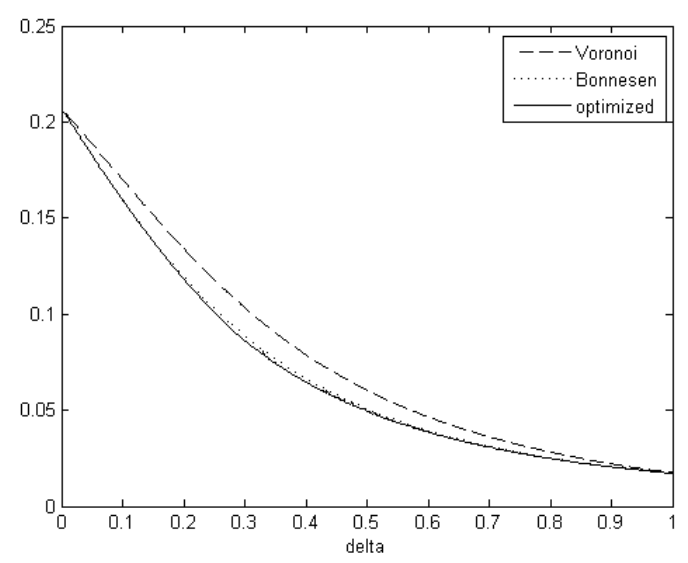

Fig. 5. Thickness of the minimal annulus for 16 directional vectors on a rectangular grid in $2 D$ depending on $\zeta_{1}$ for Voronoi, Bonnesen, and optimized weights, respectively (see the text for details).

It is easy to see that in the case of a square grid $\left(\zeta_{1}=1\right)$ the width $R-r$ of the minimal annulus is minimal if and only if all weights are equal, $c_{1}=$ $\ldots=c_{8}=1 / 8$, a choice which coincides with the Voronoi and the Bonnesen weights. The corresponding zonotope $Z$ is a regular octagon with side length $1 / 2$ and facets parallel to the vectors $v_{1}, \ldots, v_{8} . Z$ has circumradius $R=\sqrt{4+2 \sqrt{2}} / 4$ and inradius $r=$ $(1+\sqrt{2}) / 4$. With $\gamma_{2}=2 / \pi$ we get

$$
\begin{aligned}
0.948 & \approx \frac{\pi}{8}(1+\sqrt{2}) \leq \lim _{t \searrow 0} \frac{\mathbb{E} \widehat{S}_{8}^{2}(A ; t)}{S(A)} \\
& \leq \frac{\pi}{8} \sqrt{4+2 \sqrt{2}} \approx 1.026 .
\end{aligned}
$$

This was also obtained in Kiderlen and Jensen (2003).
We consider also the case $n=3$ in the plane, i.e., we use all 16 directions obtained by normalizing the grid points in the cuboid $\left[-2 \zeta_{1}, 2 \zeta_{1}\right] \times[-2,2] \backslash\{0\}$. Now even in the special case when $\zeta_{1}=1$ the 16 weights leading to a shortest asymptotic error interval are not equal. We refrain from explicitly stating the formulas for the in- and circumradii because of their complexity. The results are qualitatively similar to the case $n=2$. The use of the Bonnesen weights yields a smaller thickness of the minimal annulus than the use of the Voronoi weights. The minimal thickness achieved with optimized weights is only slightly better than for Bonnesen weights (Fig. 5).

\section{THE THREE-DIMENSIONAL CASE}

In three dimensions, we restrict considerations to the cubic grid $\left(\zeta_{1}=\zeta_{2}=1\right)$ and $n=2$. The directions associated to the $k=3^{3}-1=26$ grid points $v_{i}$ of the set in Eq. 29 consist of the 6 directions along the edges $(i=1, \ldots, 6), 12$ diagonals of the faces $(i=7, \ldots, 18)$ and 8 spatial diagonals $(i=19, \ldots, 26)$ of the unit cube $[0,1]^{3}$. Hence, the surface area estimator in Eq. 28 is based on comparison of pixels with neighbors in the so-called 26-neighborhood. The Voronoi weights can be calculated as the relative sizes of the Voronoi cells on the unit sphere generated by $\left\{v_{1} /\left\|v_{1}\right\|, \ldots, v_{k} /\left\|v_{k}\right\|\right\}$. They can be derived by computing the area of the Voronoi cells on the unit sphere analytically with the help of spherical trigonometry, and are given by

$$
c_{i}=\left\{\begin{aligned}
\frac{1}{2}-\frac{2}{\pi} \arccos \left(\frac{\sqrt{2}+\sqrt{3}}{\sqrt{2} \sqrt{3-\sqrt{3}}} \sin \left(\frac{\pi}{8}\right)\right) & \approx 0.0457779, \\
& \text { if } i=1, \ldots, 6, \\
\frac{1}{2}-\frac{1}{\pi} \arccos \left(\frac{\sqrt{6}-2}{2 \sqrt{3-\sqrt{6}}} \sin \left(\frac{\pi}{8}\right)\right) & \approx 0.0369806, \\
& \text { if } i=7, \ldots, 18, \\
\frac{1}{2}-\frac{3}{2 \pi} \arccos \left(\frac{(2-\sqrt{3})(2-\sqrt{6})+2}{4 \sqrt{3-\sqrt{3}} \sqrt{3-\sqrt{6}}}\right) & \approx 0.0351956, \\
& \text { if } i=19, \ldots, 26 .
\end{aligned}\right.
$$

The zonotope $Z$ is the convex hull of all points of the form $\sum_{i=1}^{26} \varepsilon_{i} v_{i} /\left\|v_{i}\right\|$, where $\left(\varepsilon_{1}, \ldots, \varepsilon_{26}\right)$ runs through all vectors in $\{-1,0,1\}^{26}$. The quickhull-algorithm (Barber et al., 1996) was used to find this convex hull. $Z$ has 96 vertices, inradius $r_{\text {Vor }} \approx 0.463312$, circumradius $R_{\text {Vor }} \approx 0.511386$, and thickness of minimal annulus $T_{\text {Vor }} \approx 0.0480748$. As $\gamma_{3}=1 / 2$ we obtain the bounds

$$
0.927 \leq \lim _{t \searrow 0} \frac{\mathbb{E} \widehat{S}_{26}^{3}(A ; t)}{S(A)} \leq 1.023 .
$$

Although the definition of the Bonnesen weights was restricted to the planar case, it can naturally 
be generalized to higher dimensions. We will do so for comparison with the established Voronoi weights. Recall the construction for the Bonnesen weights in the plane for given vectors $v_{1}, \ldots, v_{k} \in \mathbb{R}^{2} \backslash\{o\}$. We defined $u_{i}=v_{i} /\left\|v_{i}\right\|, i=1, \ldots, k$, and constructed the polygon $\widetilde{P}$ in Eq. 15 with outer unit vectors $\pm u_{1}, \ldots, \pm u_{k}$ circumscribing the unit ball. We then chose $\widetilde{c_{i}}$ proportional to the length of the edge of $\widetilde{P}$ with outer unit normal $u_{i}$. In higher dimensions, for given $v_{1}, \ldots, v_{k} \in \mathbb{R}^{d} \backslash\{o\}$, we set $u_{i}=v_{i} /\left\|v_{i}\right\|, i=1, \ldots, k$, and

$$
\widetilde{P}:=\bigcap_{i=1}^{k}\left\{x \in \mathbb{R}^{d}:\left|\left\langle u_{i}, x\right\rangle\right| \leq 1\right\},
$$

in complete analogy to Eq. 15 . Hence $\widetilde{P}$ is the polytope circumscribing the unit ball with facet normals in $\left\{ \pm u_{1}, \ldots, \pm u_{k}\right\}$. We then choose $\widetilde{c}_{i}$ proportional to the $(d-1)$-dimensional volume of the facet of $\widetilde{P}$ which has outer unit normal $u_{i}$. In the present threedimensional example (with $\zeta_{1}=\zeta_{2}=1$ and all grid points in $\mathscr{V}_{2}^{(3)}$ ) the Bonnesen weights were computed with quickhull and are given by

$$
\widetilde{c}_{i} \approx \begin{cases}0.0465894, & \text { if } i=1, \ldots, 6 \\ 0.0367439, & \text { if } i=7, \ldots, 18 \\ 0.0349421, & \text { if } i=19, \ldots 26\end{cases}
$$

The associated inradius is $r_{\mathrm{Bon}} \approx 0.462424$, the circumradius is $R_{\mathrm{Bon}} \approx 0.511243$, and thickness of minimal annulus is $T_{\mathrm{Bon}} \approx 0.0488187$. This shows that Bonnesen weights are not better than Voronoi weights for the $3^{d}-1$ directions in dimension $d=3$. But this is not surprising because they are based on Bonnesen's inequality Eq. 14 which is valid only for two-dimensional convex bodies. In the last section we discuss how the approach developed in this paper could be extended to higher dimensions.

Finally, we show how the asymptotic relative error bounds in the case of a quadratic $\left(\zeta_{1}=1\right)$ planar grid depend on the choice of $n$. To do so, the symmetrized spread of the normalized vectors of $\mathscr{V}_{n}^{(2)}$ must be determined.

Lemma 6 For $n \geq 2$ the symmetrized spread of $\left\{x /\|x\|: x \in \mathscr{V}_{n}^{(2)}\right\}$ is equal to

$$
d_{n}=\sqrt{2-\sqrt{2\left(1+\frac{n-1}{\sqrt{1+(n-1)^{2}}}\right)}} \leq \frac{1}{2(n-1)} .
$$

Proof Let $\Delta$ be the symmetrized spread of $D:=$ $\left\{x /\|x\|: x \in \mathscr{V}_{n}^{(2)}\right\}$ and set $m:=n-1$. Clearly, the arc $C \subset \mathbb{S}^{1}$ in the first quadrant with endpoints $(1,0)^{\top},\left(m^{2}+1\right)^{-1 / 2}(m, 1)^{\top} \in D$ does not contain any other points in $D$ and thus the spread $\Delta$ is at least the distance of the midpoint of $C$ to one of its endpoints. Hence

$\Delta \geq \sqrt{2\left(1-\cos \frac{\varphi}{2}\right)}=\sqrt{2\left(1-\sqrt{\frac{1+\cos \varphi}{2}}\right)}=d_{n}$,

where $\varphi=\arccos \left(m / \sqrt{1+m^{2}}\right)$ is the length of $C$. This interpretation of $d_{n}$ also shows the inequality in Eq. 30, as $d_{n}$ cannot be larger then half the distance of $(1,0)^{\top}$ from $(1,1 / m)^{\top}$. To show that $\Delta \leq d_{n}$, let $v, v^{\prime} \in D$ two points such that the sub-arc of $\mathbb{S}^{1}$ connecting them does not contain any other points of $D$. Using reflections and translations leaving $\mathbb{Z}^{2}$ invariant, we may assume that $v=x /\|x\|$ and $v^{\prime}=$ $x^{\prime} /\left\|x^{\prime}\right\|$ where $x, x^{\prime} \in \mathscr{V}_{n}^{(2)}$ and the angles they form with the $\mathrm{x}$-axis are at most $\pi / 4$. We refer to Fig. 6 for a sketch of the situation. The cone between the rays spanned by $x$ and $x^{\prime}$ cannot contain any other points of $\mathscr{V}_{n}{ }^{(2)}$ in its interior. Let $y\left(y^{\prime}\right)$ denote the point in $\mathscr{V}_{n}^{(2)} \cap\{(m, t): t \geq 0\}$ with largest (smallest) second coordinate below (above) this cone. Then the length of the arc in $\mathbb{S}^{1}$ with endpoints $v$ and $v^{\prime}$ is at most the length of the arc $C^{\prime} \subset \mathbb{S}^{1}$ with endpoints $y /\|y\|$ and $y^{\prime} /\left\|y^{\prime}\right\|$. The segment $\left[y, y^{\prime}\right]$ does not contain any points of $\mathscr{V}_{n}^{(2)}$, so $y$ and $y^{\prime}$ are distance one apart, and the length of $C^{\prime}$ is bounded from above by the length of $C$. Here we use the fact that among all unit intervals in $\left\{(m, t)^{\top}: t \geq 0\right\}$, the interval $\left[(m, 0)^{\top},(m, 1)^{\top}\right]$ has the largest gnomonic projection. This gives $\Delta \leq d_{n}$, as $v$ and $v^{\prime}$ where arbitrary in $D$.

In view of Theorem 4, Lemma 6 yields an estimate for the asymptotic relative error using the Bonnesenweights $c_{i}=\widetilde{c}_{i}$ whenever $n \geq 2$.

Theorem 7 Let $A \subset \mathbb{R}^{2}$ be a topologically regular polyconvex set with positive perimeter, $n \geq 2$, and $\zeta_{1}=1$. Let $\widehat{S}_{k}^{2}(A ; t)$ be defined by Eq. 28 , where $\left\{v_{1}, \ldots, v_{k}\right\}=\mathscr{V}_{n}^{(2)}$ and $c_{i}=\widetilde{c}_{i}, i=1, \ldots, k$, are the Bonnesen weights. Then the asymptotic relative mean error obeys

$$
\lim _{t \searrow 0}\left|\frac{\mathbb{E} \widehat{S}_{k}^{2}(A ; t)-S(A)}{S(A)}\right| \leq \frac{\pi^{2}}{12}(n-1)^{-2} .
$$




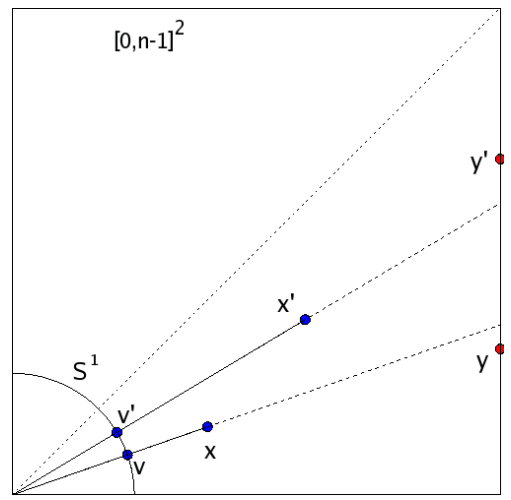

Fig. 6. Construction to determine the symmetrized spread in Lemma 6: the points $x$ and $x^{\prime}$ in $\mathscr{V}_{n}^{(2)}$ are contained in the cube $[0, n-1]^{2}$ and their normalizations $v, v^{\prime}$ in $\mathbb{S}^{1}$.

\section{EXTENSION TO HIGHER DIMENSIONS}

Large parts of the present worst case analysis for quadrature rules, including the use of an associated zonotope $Z$, are not restricted to the two-dimensional setting. In order to find an easily accessible upper bound for the width of the minimal annulus, a joint extension of Bonnesen's refined isoperimetric inequalities Eq. 14 and the geometric inequality of Groemer (1990) to higher dimensions (which is known) is not suitable, as it involves the surface area of $Z$. Instead, a strengthened version of Uhrysohn's inequality is appropriate. It reads

$$
\left(\frac{b(Z)}{b\left(B^{d}\right)}\right)^{d}-\frac{V_{d}(Z)}{V_{d}\left(B^{d}\right)} \geq c_{d}(Z) \delta_{2}^{2}\left(Z, \gamma_{d} B^{d}\right) .
$$

Here $c_{d}$ is an explicitly known constant, depending on $d$, the mean width $b(Z)$ of $Z$, and the second intrinsic volume of $Z$. This inequality is a special case of a whole family of geometric inequalities derived by Groemer and Schneider (1991), who also showed that it implies the Bonnesen type inequality

$$
\left(\frac{b(Z)}{b\left(B^{d}\right)}\right)^{d}-\frac{V_{d}(Z)}{V_{d}\left(B^{d}\right)} \geq c_{d}^{\prime}(Z)(R-r)^{(d+3) / 2} .
$$

The constant $c_{d}^{\prime}$, again, depends on $d, b(Z)$ and the second intrinsic volume of $Z$. For $d=2$ Ineq. 32 is of the same form as Ineq. 14, but with a weaker exponent. This apparently suboptimal exponent, and the problem to determine the zonotope with given mean width which minimizes the left hand side of Eq. 32 for $d>2$, limits the usefulness of these inequalities for applications.

\section{ACKNOWLEDGMENTS}

We want to thank Luis Cruz-Orive, Maria Hernandez Cifre, Ilya Molchanov, and the two anonymous referees for their helpful comments on an earlier version of this paper. The first author's work was partially supported by the Carlsberg foundation and the Danish Council for Strategic Research. The second author's work was partially supported by a Marie Curie Fellowship of the European Community Programme.

\section{REFERENCES}

Barber C, Dobkin D, Huhdanpaa H (1996). The Quickhull algorithm for convex hulls. ACM Trans Math Soft 22:469-83.

Bonnesen T (1929). Problèmes des Isopérimètres et des Isépiphanes. Paris: Gauthier-Villars.

Gardner R, Kiderlen M, Milanfar P (2006). Convergence of algorithms for reconstructing convex bodies and directional measures. Ann Stat 34:1331-74.

Goodey P, Weil W (1993). Zonoids and generalizations. In: Gruber P, Wills J, eds., Handbook of Convex Geometry, vol. B. Amsterdam: Elsevier, 1297-326.

Groemer H (1990). Stability properties of geometric inequalities. Am Math Mon 97:382-94.

Groemer H, Schneider R (1991). Stability estimates for some geometric inequalities. Bull Lond Math Soc 23:67-74.

Gruber P (2004). Optimum quantization and its applications. Adv Math 186:456-97.

Janáček J (2001). Estimating length and surface area by systematic projections. Image Anal Stereol 20 (Suppl. 1):89-94.

Kiderlen M, Jensen E (2003). Estimation of the directional measure of planar random sets by digitization. Adv Appl Probab 35:583-602.

Kiderlen M, Rataj J (2006). On infinitesimal increase of volumes of morphological transforms. Mathematika 53:103-27.

Knebelman M (1941). Two isoperimetric problems. Am Math Mon 48:623-7.

Lindelöf L (1869). Propriétés générales des polyèdres qui, sous une étendue superficielle donnée, renferment le plus grand volume. Bull Acad Sci St Petersburg 14:25869. Math Ann (1870) 2:150-159.

Moran P (1966). Measuring the length of a curve. Biometrika 53:359-64. 
Ohser J, Mücklich F (2000). Statistical Analysis of Microstructures in Material Science. Chichester: John Wiley \& Sons.

Rataj J (2002). Determination of spherical area measure by means of dilation volumes. Math Nachr 235:143-62.

Rother W, Zähle M (1990). A short proof of a principal kinematic formula and extensions. Trans Amer Math Soc 321:547-58.
Schneider R (1993). Convex Bodies: The Brunn-Minkowski Theory, vol. 44 of Encyclopedia of Mathematics and its Applications. Cambridge: Cambridge University Press.

Schneider R, Weil W (1992). Integralgeometrie. Stuttgart: Teubner.

Steinhaus H (1930). Praxis der Rektifikation und zum Längenbegriff. Berich Sachs Akad Wiss Leipzig 82:120-30. 